\title{
Physical properties of galaxies and their evolution in the VIMOS VLT Deep Survey ${ }^{\star}$
}

\section{Extending the mass-metallicity relation to the range $z \approx 0.89-1.24$}

\author{
E. Pérez-Montero ${ }^{1, \star \star}$, T. Contini ${ }^{1}$, F. Lamareille ${ }^{1}$, J. Brinchmann ${ }^{19}$, C. J. Walcher ${ }^{7}$, S. Charlot ${ }^{8,10}$, M. Bolzonella ${ }^{3}$,
} L. Pozzetti $^{3}$, D. Bottini ${ }^{2}$, B. Garilli ${ }^{2}$, V. Le Brun ${ }^{7}$, O. Le Fèvre ${ }^{7}$, D. Maccagni ${ }^{2}$, R. Scaramella ${ }^{4,13}$, M. Scodeggio ${ }^{2}$, L. Tresse ${ }^{7}$, G. Vettolani ${ }^{4}$, A. Zanichelli ${ }^{4}$, C. Adami ${ }^{7}$, S. Arnouts ${ }^{23,7}$, S. Bardelli ${ }^{3}$, A. Cappi ${ }^{3}$, P. Ciliegi ${ }^{3}$, S. Foucaud $^{21}$, P. Franzetti ${ }^{2}$, I. Gavignaud ${ }^{12}$, L. Guzzo 9 , O. Ilbert ${ }^{20}$, A. Iovino ${ }^{9}$, H. J. McCracken ${ }^{10,11}$, B. Marano ${ }^{6}$, C. Marinoni ${ }^{18}$, A. Mazure $^{7}$, B. Meneux ${ }^{2,9}$, R. Merighi ${ }^{3}$, S. Paltani ${ }^{15,16}$, R. Pellò ${ }^{1}$, A. Pollo ${ }^{7,17}$, M. Radovich ${ }^{5}$, D. Vergani ${ }^{2}$, G. Zamorani ${ }^{3}$, and E. Zucca ${ }^{3}$

(Affiliations can be found after the references)

Received 10 July 2008 / Accepted 3 October 2008

\section{ABSTRACT}

Aims. We present a continuation of our study about the relation between stellar mass and gas-phase metallicity in the VIMOS VLT Deep Survey (VVDS). In this work we extend the determination of metallicities up to redshift $\approx 1.24$ for a sample of 42 star-forming galaxies with a mean redshift value of 0.99 .

Methods. For a selected sample of emission-line galaxies, we use both diagnostic diagrams and empirical calibrations based on [OII] emission lines along with the empirical relation between the intensities of the [OIII] and [NeIII] emission lines and the theoretical ratios between Balmer recombination emission lines to identify star-forming galaxies and to derive their metallicities. We derive stellar masses by fitting the whole spectral energy distribution with a set of stellar population synthesis models.

Results. These new methods allow us to extend the mass-metallicity relation to higher redshift. We show that the metallicity determinations are consistent with more established strong-line methods. Taken together this allows us to study the evolution of the mass-metallicity relation up to $z \approx 1.24$ with good control of systematic uncertainties. We find an evolution with redshift of the average metallicity of galaxies very similar to those reported in the literature: for a given stellar mass, galaxies at $z \sim 1$ have, on average, a metallicity $\sim 0.3$ dex lower than galaxies in the local universe. However we do not see any significant metallicity evolution between redshifts $z \sim 0.7$ (Paper I) and $z \sim 1.0$ (this paper). We find also the same flattening of the mass-metallicity relation for the most massive galaxies as reported in Paper I at lower redshifts, but again no apparent evolution of the slope is seen between $z \sim 0.7$ and $z \sim 1.0$.

Key words. galaxies: evolution - galaxies: fundamental parameters - galaxies: abundances - galaxies: starburst

\section{Introduction}

The formation and evolution of galaxies at different cosmological epochs are driven mainly by two processes: the star formation history and the metal enrichment. These processes, which are strongly connected in the evolution of individual galaxies, can be altered by the peculiar dynamics of the gas. The rate of star formation can be increased as a consequence of mergers or due to the inflow of gas. Regarding metallicity, it is sensitive to metal losses due to stellar winds, supernovae and active nucleus feedbacks. Therefore, the study of the dominant mechanisms of evolution of galaxies at different redshifts rely on the determination of metallicity and total stellar mass.

The correlation between stellar mass and metallicity (M-Z relation) was firstly found in the nearby universe by Lequeux et al. (1979) for dwarf irregular galaxies. Since them,

\footnotetext{
* Based on data obtained with the European Southern Observatory Very Large Telescope, Paranal, Chile, program 070.A-9007, and on data obtained at the Canada-France-Hawaii Telescope, operated by the CNRS in France, CNRC in Canada and the University of Hawaii.

$\star \star$ Postdoctoral fellow of the Spanish Fundación Española para la Ciencia y la Tecnología.
}

numerous works have been performed in order to understand and quantify this relation in the local universe (among others Skillman et al. 1989; Brodie \& Huchra 1991; Zaritsky et al. 1994; Richer \& McCall 1994; Garnett et al. 1997; or Pilyugin \& Ferrini 2000). This relation has its counterpart in the luminositymetallicity relation which is easier to derive when a lower number of spectrophotometric bands are available. Besides, in the last years, this work has been complemented with the use of large surveys of galaxies which allow the derivation of this relation in samples statistically more significant, (e.g. SDSS, Tremonti et al. 2004; 2dFGRS, Lamareille et al. 2004), that confirm the decrease of metal loss when galaxy stellar mass increases. Several studies of the evolution of this relation at larger redshifts have appeared due to the growing number of spectroscopic surveys of star-forming galaxies in the distant universe. This is the case of Savaglio et al. (2005) or Lamareille et al. (2006) who agree to find lower mean metallicities for the same galaxy mass bins at larger redshifts. However, they do not agree about the evolution of the slope of the M-Z relation: Lamareille et al. (2006) reported a flattening of the relation at higher redshifts whereas Savaglio et al. (2005) found a steepening of the $\mathrm{M}-\mathrm{Z}$ relation. Unfortunately, these studies are limited to $z<1$ 
galaxies, due to the required presence of certain emission lines in the optical spectrum to derive the metallicity. Erb et al. (2006) studied this relation for a sample of star forming galaxies at $z \approx 2$ using near-infrared spectra and they find a lower value of the mean metallicity for a given galaxy stellar mass. This evolution is apparently much more stronger at higher redshift as reported by Maiolino et al. (2008), using also IR spectra for a sample of star-forming galaxies ar $z>3$. However, there is a lack of observations and available data in the redshift range $0.9-2$, corresponding to the epoch of the universe when the maximum of star formation took place. It is therefore particularly interesting to study the $\mathrm{M}-\mathrm{Z}$ relation at this period.

The estimation of the global trend of the $\mathrm{M}-\mathrm{Z}$ relation at different cosmological epochs is possible only through the analysis of statistically significant samples with accurate determinations of the stellar mass and the gas phase oxygen abundance. This paper is a continuation of Lamareille et al. (2008, hereinafter Paper I), who study the M-Z relation of the star-forming galaxies detected in the VIMOS VLT Deep Survey up to redshift 0.89. In this work we extend the upper redshift limit of the study up to $z \approx 1.24$, using the relation between metallicity and emissionline strength of [OII] and [NeIII] presented in Pérez-Montero et al. (2007). In the next section we describe the sample of starforming galaxies discussing the selection criteria and the methods to derive their stellar mass and metal content, which can go to higher redshift than other classical methods. In Sect. 3, we describe the M-Z relation in the redshift bin $0.89<z<1.24$, with an average value in our sample of $z=0.99$ and we compare our results with those found in Paper I. Finally, we summarise our results and present our conclusions in the last section. Throughout this paper we normalize the derived stellar masses and the absolute magnitudes with the standard $\Lambda$-CDM cosmology, i.e., $h=0.7, \Omega_{\mathrm{m}}=0.3$ and $\Omega_{\Lambda}=0.7$ (Spergel et al. 2007).

\section{Description of the sample}

The VIMOS/VLT Deep Survey (VVDS, Le Fèvre et al. 2005) is one of the widest and deepest spectrophotometric surveys of distant galaxies with a mean redshift of $z \approx 0.7$. The optical spectroscopic data belong to the first epoch sample obtained with the VIsible MultiObject Spectrograph (VIMOS) at ESO/VLT (UT3). They are divided in two deep fields $\left(17.5 \leq I_{\mathrm{AB}} \leq 24\right)$ : VVDS-02h (hereafter F02; Le Fèvre et al. 2005) and CDFS (Le Fèvre et al. 2004) and in three wide fields $\left(17.5 \leq I_{\mathrm{AB}} \leq\right.$ 22.5): VVDS-10h, VVDS-14h and VVDS-22h (hereafter F10, F14 and F22 respectively, Garilli et al., in prep.). As explained in Paper I, we divide therefore our total sample in two: a deep one with the F02 and CDFS fields and a wide one with F10, F14 and F22 fields. Spectroscopic data have been reduced using the VIPGI pipeline (Scodeggio et al. 2005).

The following selection criteria have been applied on this sample. We consider only objects with a redshift known at a $75 \%$ confidence level (i.e. VVDS redshift flags 2, 3, 4 and 9) and we remove all stars, broad-line Active Galactic Nuclei (hereafter AGNs, Gavignaud et al. 2006) and duplicate observations.

The optical spectra of each galaxy have been split into two components (stellar and nebular) using the pipeline platefit_vimos as described in Paper I. This software removes the stellar continuum and absorption lines and then fits automatically all emission lines. The templates used to fit the stellar component have been derived from the libraries of Bruzual \& Charlot (2003) resampled to the velocity dispersion of VVDS spectra. The fitting and measurement procedures of all emission lines (flux and equivalent width) and stellar indices in the original spectra are

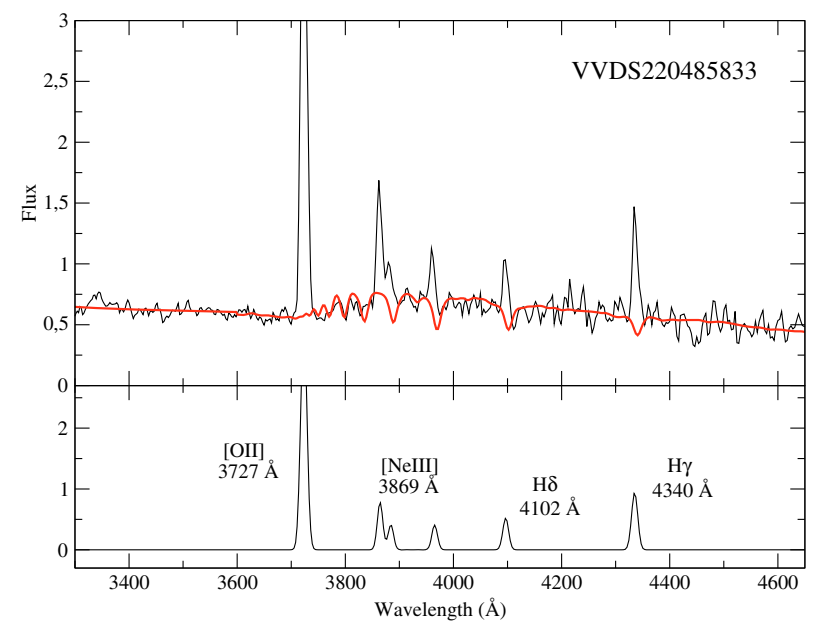

Fig. 1. VIMOS rest-frame spectrum of the galaxy VVDS220485833. The top panel illustrates the fitting of the stellar continuum obtained with the platefit_vimos tool. In the bottom panel, we show the resultant nebular emission-lines spectrum, once the fitted stellar continuum has been substracted. The labels indicate the set of emission lines used in this work to derive metallicities.

described in Paper I. In Fig. 1 we show an example of the fitting of the stellar continuum (top panel) made by platefit_vimos for the galaxy VVDS221485833, one of the best S/N spectra. The resultant nebular emission-lines spectrum, once this fitting is substracted, is shown in the bottom panel.

\subsection{Selection of star-forming galaxies}

In order to correctly study the M-Z relation, we select the galaxies whose main mechanism of ionization is star formation. We use emission-line diagnostic diagrams to identify narrow-line AGNs (Seyfert 2 and LINERS) once broad line AGNs have been removed. In particular we use the "blue" diagnostic diagram based on [OIII] $5007 \AA, \mathrm{H} \beta$ and [OII] $3727 \AA$ emission lines (Lamareille et al. 2004) and applied in Paper I for the redshift bin $0.5<z<0.9$. We extend this analysis up to redshift $\sim 1.3 \mathrm{using}$ the theoretical ratios between $\mathrm{H} \beta$ and the most intense Balmer hydrogen recombination lines at bluer wavelengths. This is the case of $\mathrm{H} \gamma$ at $4340 \AA$ and $\mathrm{H} \delta$ at $4102 \AA$. We assume the following values for these ratios, typical of star-forming galaxies: $\mathrm{H} \gamma / \mathrm{H} \beta=0.48$ and $\mathrm{H} \delta / \mathrm{H} \beta=0.25$.

Although these ratios slightly depend on the electron temperature of the ionized gas, this dependence is negligible in comparison with the intrinsic uncertainty of the measurement of the involved emission lines, even in the case of narrowline AGNs, which show the lowest values of these ratios. We apply the empirical relation found between the emission-line ratio of [OIII]4959+5007 $\AA$ and [NeIII]3869 $\AA$ (Pérez-Montero et al. 2007). The mean value of this ratio reported in this study for a sample of HII regions of the local universe is equal to 15.37. However, the mean value of the same ratio for the VVDS star-forming galaxies (both for the deep and wide fields) is equal to 17.37. We correct this slight difference taking into account a reddening correction corresponding to an extinction of $A_{V}=0.5 \mathrm{mag}$. After this correction, the mean value of [OIII]4959+5007 $\AA /[\mathrm{NeIII}] 3869 \AA$ emission-line ratio for the VVDS galaxies is 15.23 , which is almost identical to the value found for the reddening corrected HII regions of the local universe. Therefore, we decide to use the same reddening correction for the rest of the calculations in the VVDS sample. 


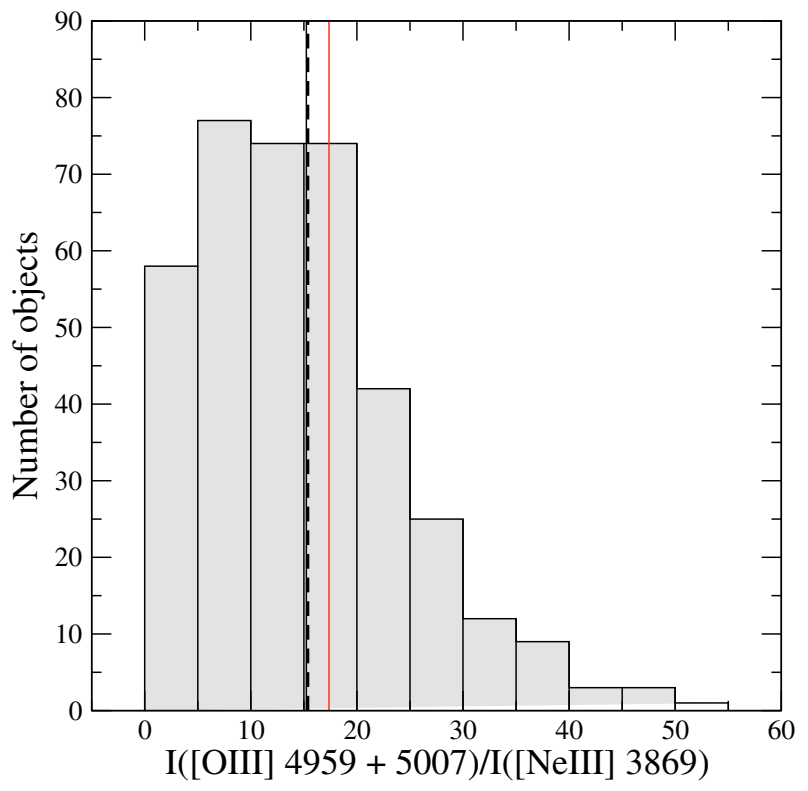

Fig. 2. Histogram of the distribution of the ratio between the deredenned fluxes of [OIII]4959+5007 $\AA$ and [NeIII]3869 $\AA$ emission lines for the five VVDS fields (VVDS-02h, VVDS-10h, VVDS-14h, VVDS$22 \mathrm{~h}$ and CDFS). The mean value of this distribution, shown as a black solid line, is 15.23. The red solid line represents the mean value of the distribution without applying the mean reddening correction and the dashed line is mean value $(=15.37)$ of this emission-line ratio for a representative sample of nearby galaxies studied in Pérez-Montero et al. (2007).

In Fig. 2, we show the distribution of the emission-line ratio [OIII]4959+5007 $/ /[\mathrm{NeIII}] 3869 \AA$ for the 379 VVDS secure and candidates star-forming galaxies for which we have measured these lines in the redshift range $0.5<z<0.9$ and with an error on the ratio lower than the ratio itself. In this figure we compare the mean value of this distribution, once extinction corrected (black solid line), the mean value found without extinction correction (red solid line) and the value found in the local universe by Pérez-Montero et al. (2007, dashed line).

The empirical limit between star-forming galaxies and narrow-line AGNs in the "blue-Ne" diagnostic diagram based on [NeIII] $3869 \AA$ emission line takes the following form:

$\log \left(\frac{[\mathrm{NeIII}]}{\mathrm{H} \beta}\right)=\frac{0.14}{\log ([\mathrm{OII}] / \mathrm{H} \beta)-1.45}-0.23$.

Following the same prescriptions as in the "blue" diagnostic method, the objects lying under this line are considered as starforming galaxies, while those lying above it are considered as AGNs. This diagnostic diagram is, in fact, equivalent to that proposed by Rola et al. (1997) which is based on the same emission lines. In the AGN zone, LINERs are identified by the following criterium:

$\log \left(\frac{I([\mathrm{NeIII}] 3869}{I(\mathrm{H} \beta)}\right)<-0.57$.

Adopting the emission-line ratios $\mathrm{H} \gamma / \mathrm{H} \beta$ and $\mathrm{H} \delta / \mathrm{H} \beta$ listed above, we can use the same expression for galaxies at redshifts higher than 0.9 or for those whose $\mathrm{H} \beta$ emission line is not measured in the redshift range $0.5<z<0.9$. In the case of $\mathrm{H} \delta$, these expressions take the same form as the Eqs. (23) and (24) in Pérez-Montero et al. (2007).

In Fig. 3 we show the $[\mathrm{OII}] / \mathrm{H} \beta$ vs. $[\mathrm{NeIII}] / \mathrm{H} \beta$ relation for the sample of VVDS galaxies with the appropriate lines, in the redshift ranges $0.5<z<0.9$ (left panel) and $0.9<z<1.24$ (right panel). The dashed lines delineate the uncertainty domain ( \pm 0.15 dex around the empirical limit) in the spectral classification: objects lying in this region are considered as "candidates" to star-forming galaxies. The results of this analysis are summarised in Table 1 . We make also a comparison with the classification derived from the "blue" diagnostic diagram based on [OII] and [OIII] emission lines for galaxies in the redshift range $0.5<z<0.9$.

We see that the fraction of galaxies classified as candidates to star-forming from the diagram based on [NeIII] is much higher (at all redshift bins) that the one based on [OIII] emission line. Taking into account that the rate of coincidence between blue and blue-Ne diagnostics for the galaxies for which it is possible to apply at the same time the two methods reaches $98 \%$, we think that the sample of galaxies with accurate [NeIII] measurements (i.e. with a high enough $\mathrm{S} / \mathrm{N}$ ) is somehow biased towards a population with enhanced [NeIII] emission. This is indicated by the range of $\log ([\mathrm{NeIII}] / \mathrm{H} \beta)$ values in both panels of Fig. 3 which are between -1 and 0 , while in Fig. 3 of Paper I we see that the ratio $\log ([\mathrm{OIII}] / \mathrm{H} \beta)$, which is approximately an order of magnitude larger than $\log ([\mathrm{NeIII}] / \mathrm{H} \beta)$, varies between -0.5 and 1 . This means that in our sample we are certainly affected by some selection effect that preserves us to properly detect all the star forming galaxies with a value of $\log ([\mathrm{NeIII}] / \mathrm{H} \beta)$ between -1.5 and -1 . Therefore, the relative number of candidates is larger in this subsample.

This same effect is seen when looking at the relative number of AGNs in the redshift bin $0.5<z<0.9$, especially in the wide VVDS sample. On the other hand, when we compare the relative numbers of AGNs in the redshift regime $z>0.9$, we do not see a significant increase compared with the blue diagnostic diagram at lower redshift. Note, finally, that we did not identify any LINER in our VVDS sample.

For the following analysis of stellar masses and metallicities, we consider both the samples of candidates and secure starforming galaxies. Unfortunately, there are no deep enough X-ray or far-IR observations of the VVDS fields to check the presence of hidden AGNs.

\subsection{Estimation of stellar masses}

The procedure to derive stellar masses is based on the comparison between observations, basically the photometric Spectral Energy Distribution (hereafter SED) of galaxies, combined in some cases with their stellar absorption index $\mathrm{H} \delta$ and D4000 break measured in VIMOS spectra, and population synthesis models. As described in Paper I and introduced by SDSS collaborators (Kauffmann et al. 2003; Tremonti et al. 2004; Brinchmann et al. 2005), we are using the Bayesian approch. To summarise, the population synthesis models are based on Bruzual \& Charlot (2003), with the addition of secondary bursts to the standard declining exponential star formation history (stochastic library, Salim et al. 2005; Gallazzi et al. 2005). The SED of the models are compared with the available photometry for VVDS galaxies: CFH12k observations in BVRI bands for F02, F22 and F10 fields, and in $I$ band for F14 fields (McCracken et al. 2003; Le Fèvre et al. 2004), completed by $U$ band observations in the F02 field (Radovich et al. 2004), CFHTLS (Canada-France-Hawaii Telescope Legacy Survey) in $u^{*} g^{\prime} r^{\prime} i^{\prime} z^{\prime}$ bands for F02 and F22 fields and $J K$ s photometry available for a substantial fraction of the objects in the F02 field (Iovino et al. 2005; Temporin et al. 2006). For the CDFS field, we use CFH12k UBVRI (Arnouts et al. 2001) and HST bviz 


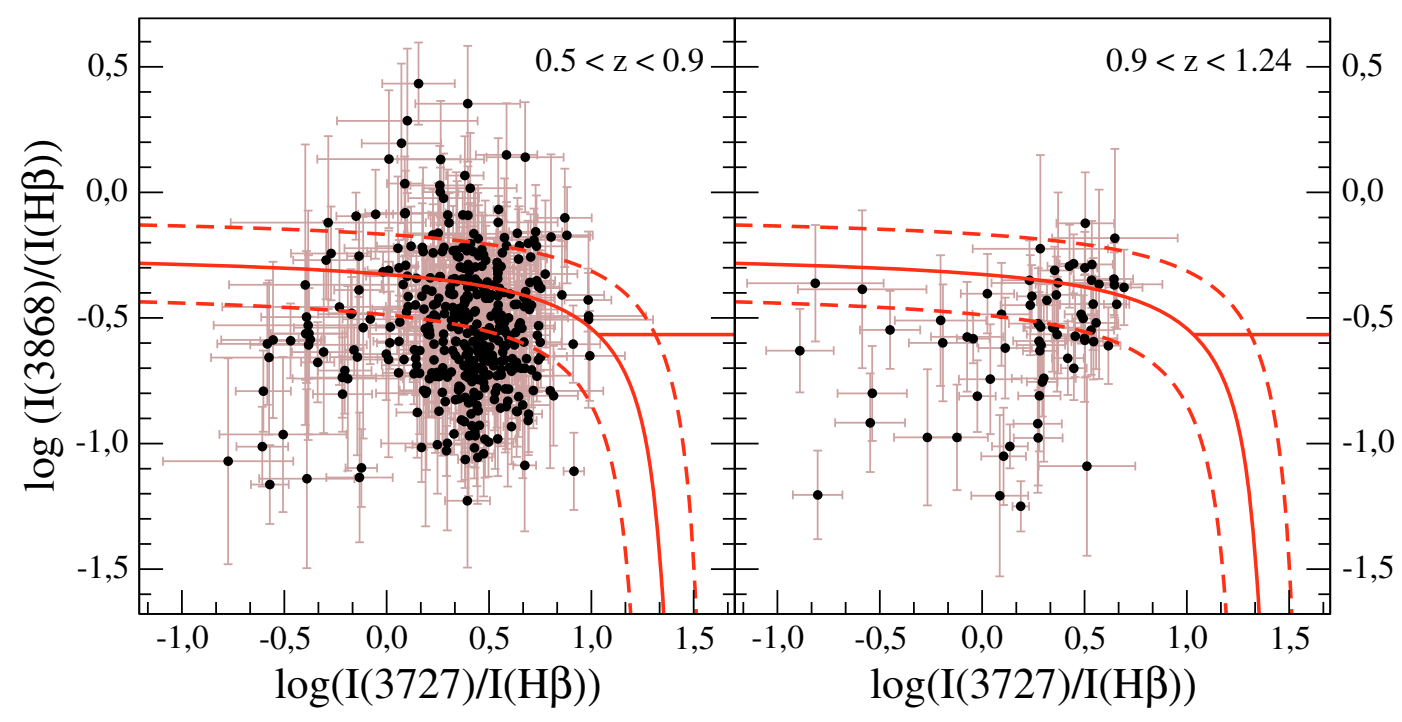

Fig. 3. Spectral classification of VVDS galaxies using the diagram diagnostic based on [NeIII] emission line. In the left panel we show the diagram for 418 emission-line galaxies in the redshift range $0.5<z<0.9$, while the right panel shows 72 emission-line galaxies in the redshift range $0.9<z<1.24$. In these two panels, the red solid curve divides the regions of star-forming galaxies and narrow-line AGNs. The straight red solid line divides the AGN region between Seyfert 2 (top) and LINER (bottom).

Table 1. Distribution of star-forming galaxies and narrow-line AGNs among VVDS emission-line galaxies using the "blue" diagnostic diagrams based on the relation between [OIII] (blue, $0.5<z<0.9$ ) or [NeIII] (blue-Ne, $0.5<z<1.24$ ) and [OII] emission lines. We show the distribution for the whole sample and for wide and deep fields. In the wide classification we include those galaxies belonging to the deep fields having $I_{\mathrm{AB}} \leq 22.5$.

\begin{tabular}{lcccccc}
\hline \hline & \multicolumn{2}{c}{$0.5<z<0.9$} & \multicolumn{2}{c}{$0.5<z<0.9$} & \multicolumn{2}{c}{$z>0.9$} \\
Sample/Diagnostic & blue & $\%$ & blue-Ne & $\%$ & blue-Ne & $\%$ \\
\hline Wide & & & & & & \\
Emission-line (total) & 924 & 100 & 338 & 100 & 62 & 100 \\
Star-forming & 768 & 83 & 157 & 45 & 34 & 55 \\
Candidates & 138 & 15 & 142 & 42 & 27 & 43 \\
Seyfert 2 & 18 & 2 & 39 & 12 & 1 & 2 \\
LINER & 0 & 0 & 0 & 0 & 0 & 0 \\
\hline Deep & & & & & & \\
Emission-line (total) & 568 & 100 & 197 & 100 & 36 & 100 \\
Star-forming & 412 & 73 & 110 & 56 & 18 & 50 \\
Candidates & 139 & 24 & 79 & 40 & 17 & 47 \\
Seyfert 2 & 17 & 3 & 8 & 4 & 1 & 3 \\
LINER & 0 & 0 & 0 & 0 & 0 & 0 \\
\hline Total & & & & & & \\
Emission-line (total) & 1213 & 100 & 418 & 100 & 72 & 100 \\
Star-forming & 945 & 78 & 216 & 52 & 40 & 55 \\
Candidates & 236 & 20 & 163 & 39 & 30 & 42 \\
Seyfert 2 & 32 & 3 & 39 & 9 & 2 & 3 \\
LINER & 0 & 0 & 0 & 0 & 0 & 0 \\
\hline
\end{tabular}

(Giavalisco et al. 2004) observations. Finally, objects in F02 and F22 fields have been cross-matched with the UKIDSS public catolog (Warren et al. 2007), providing additional observations in $J K$ bands.

As in Paper I, we correct for the fact that both minimum and maximal stellar detected masses depend on redshift. We thus define a limiting mass for each redshift bin, which is the minimum mass that would be detected at a given redshift for a given limiting apparent magnitude. For our M-Z relation study we do not consider galaxies with a stellar mass lower that this minimum mass derived in the corresponding redshift bin.

The limiting mass for the redshift range $0.89<z<1.24$ is derived empirically using the same procedure as in Paper I.
We derive the limiting mass for each galaxy following the expression:

$$
\log \left(M_{\mathrm{lim}}^{*}\right)=\log \left(M^{*}\right)+0.4 \cdot\left(I_{\mathrm{obs}}-I_{\mathrm{sel}}\right)
$$

where $M_{\lim }^{*}$ is the limiting mass in solar masses, $M^{*}$ the stellar mass in solar masses and $I_{\mathrm{obs}}$ is the observed $I$-band magnitude, $I_{\text {sel }}$ is the $I$-band magnitude limit for each field, which is equal to 24 and 22.5 in the case of deep and wide fields respectively. In Fig. 4 we show the stellar mass of the selected star-forming galaxies as a function of redshift in the range $0<z<1.24$. The solid curve shows the logarithmic fit to the $50 \%$ mass-tolight ratio completude level of the distribution of the limiting mass in each redshift bin. Since the average redshift value for the 


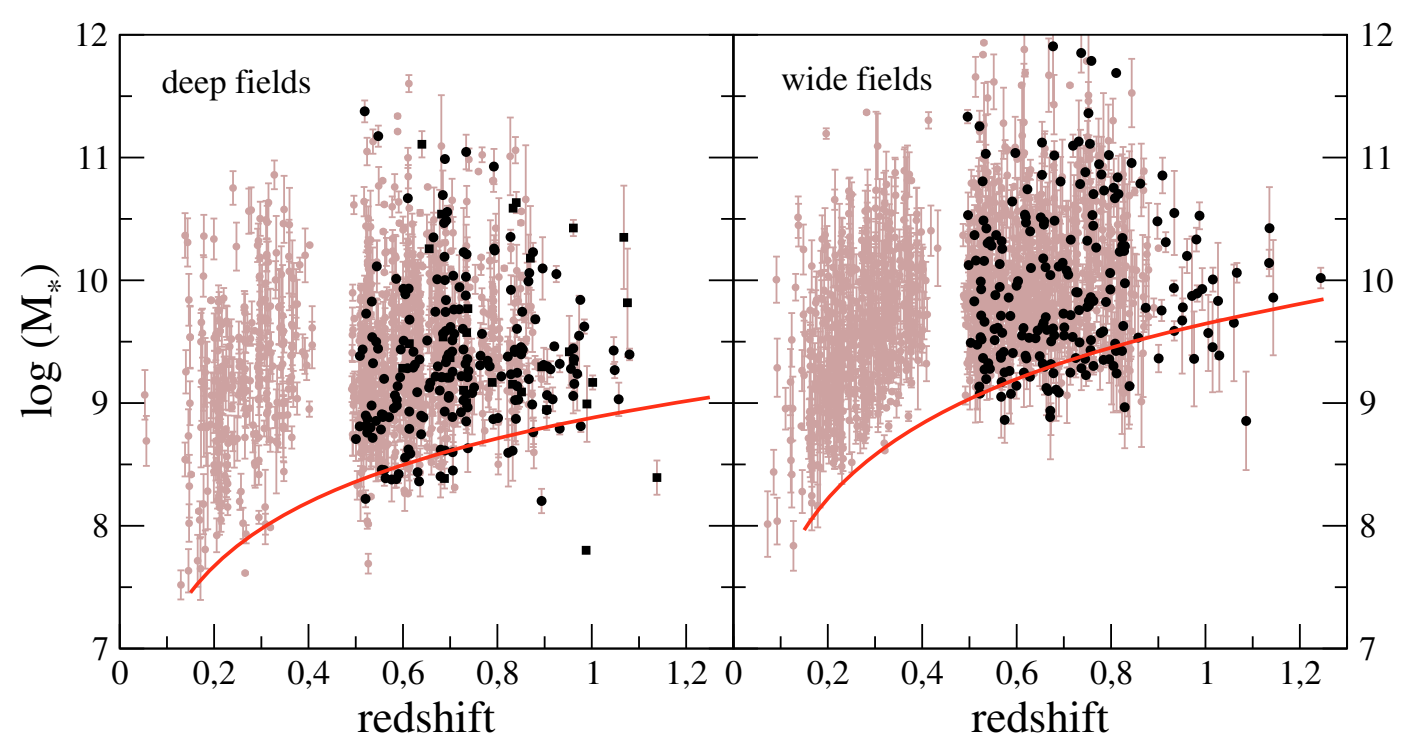

Fig. 4. Stellar masses of star-forming galaxies (in solar masses) in the VVDS deep (left panel) and wide (right panel) fields as a function of redshift. The squares represent the star-forming galaxies selected using the diagnostic diagram based on [NeIII] emission line. The solid curve represents a logarythmic fit to the 50\% mass-to-light completude levels and shows the bias imposed by the VVDS selection on apparent magnitude.

analysed galaxies in the range $0.89<z<1.24$ is $\bar{z}=1.0$, we take a value for the limiting mass in this regime of $\log \left(M_{*} / M_{\odot}\right)=8.9$ in the deep fields and 9.6 in the wide fields. As in Paper I, we obtain a lower value of the limiting mass in the deep fields. The values are also slightly larger than those derived for the starforming galaxies in the redshift bin $0.7<z<0.9$ which are 8.8 and 9.5 respectively. This is due to the fact that limiting mass increases with redshift in the selection function.

\subsection{Estimation of metallicities}

We estimate metallicities as the oxygen abundances of the ionized gas phase from the $\mathrm{O}_{2 \mathrm{Ne} 3}$ parameter (Pérez-Montero et al. 2007). This parameter is equivalent to $\mathrm{O}_{23}$ (also known as $R_{23}$ ), but it is based on the empirical relation existing between [OIII] and $[\mathrm{NeIII}]$ emission lines:

$\mathrm{O}_{2 \mathrm{Ne} 3}=\frac{I([\mathrm{OII}] 3727 \AA)+15.37 \cdot I([\mathrm{NeIII}] 3869 \AA)}{I(\mathrm{H} \beta)} \simeq \mathrm{O}_{23}$.

This calibration allows to derive metallicities using the theoretical ratios between $\mathrm{H} \beta$ and the other bright hydrogen recombination lines at bluer wavelengths, like $\mathrm{H} \gamma$ and $\mathrm{H} \delta$. It is therefore possible to derive metallicities of galaxies up to redshift $z \sim 1.25$ with the optical VIMOS spectra obtained by the VVDS. In that case, the $\mathrm{O}_{2 \mathrm{Ne} 3}$ parameter takes the following expression:

$\mathrm{O}_{2 \mathrm{Ne} 3}=\frac{I([\mathrm{OII}])+15.37 \cdot I([\mathrm{NeIII}])}{I(\mathrm{HI})} \cdot \frac{I(\mathrm{HI})}{I(\mathrm{H} \beta)}$.

We keep the factor 15.37 obtained in Pérez-Montero et al. (2007) for a sample of HII regions of the local universe once the observed fluxes have been dereddened. As shown in Fig. 2, we obtain a very similar factor to correct the flux of the involved emission lines, with a reddening equivalent to an extinction of $A_{V}=0.5 \mathrm{mag}$. This correction has a very low impact on the determination of metallicities obtained with this method: they are only 0.03 dex lower for those derived using $\mathrm{H} \gamma$ and 0.01 dex lower using $\mathrm{H} \delta$. Contrary to Paper I where equivalent widths were used as proposed by Kobulnicky \& Phillips (2003) and Lamareille et al. (2006a), emission line fluxes work better in

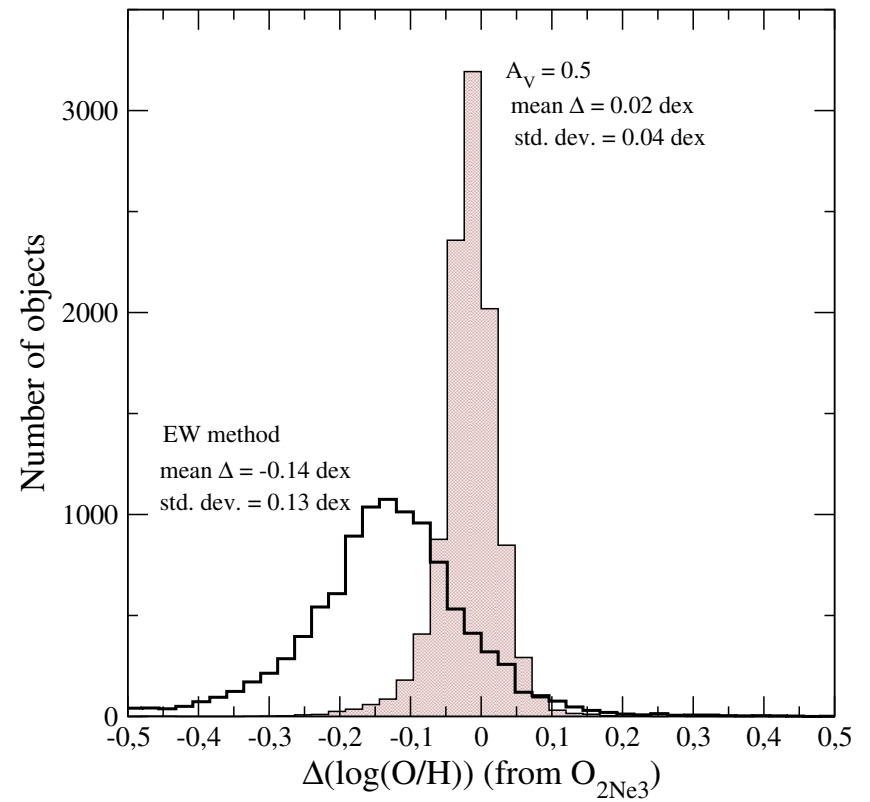

Fig. 5. Comparison between $\mathrm{O} / \mathrm{H}$ abundances derived for the star-forming galaxies of the SDSS DR4 catalogue using the $\mathrm{O}_{2 \mathrm{Ne} 3}$ parameter under different assumptions about reddening. Filled histogram represents the difference between the abundance using emission lines corrected for reddening using the Balmer decrement and assuming an average reddening corresponding to $A_{V}=0.5 \mathrm{mag}$. The thick line histogram represents the same difference but in relation with the abundances derived from the equivalent widths. We indicate also the mean value and the standard deviation of the two distributions.

the case of $\mathrm{O}_{2 \mathrm{Ne} 3}$ parameter because the variations of the continuum are more sensible to the underlying stellar population in this part of the spectrum. To illustrate this point we have done an analysis of the star-forming galaxies of the DR4 SDSS catalogue (Kauffman et al. 2003) whose involved emission lines have a signal-to-noise larger than 5. We show in Fig. 5 the histograms of the residuals to the oxygen abundances derived from $\mathrm{O}_{2 \mathrm{Ne} 3}$ and whose emission lines have been corrected for reddening using the constants derived from the Balmer decrement. 


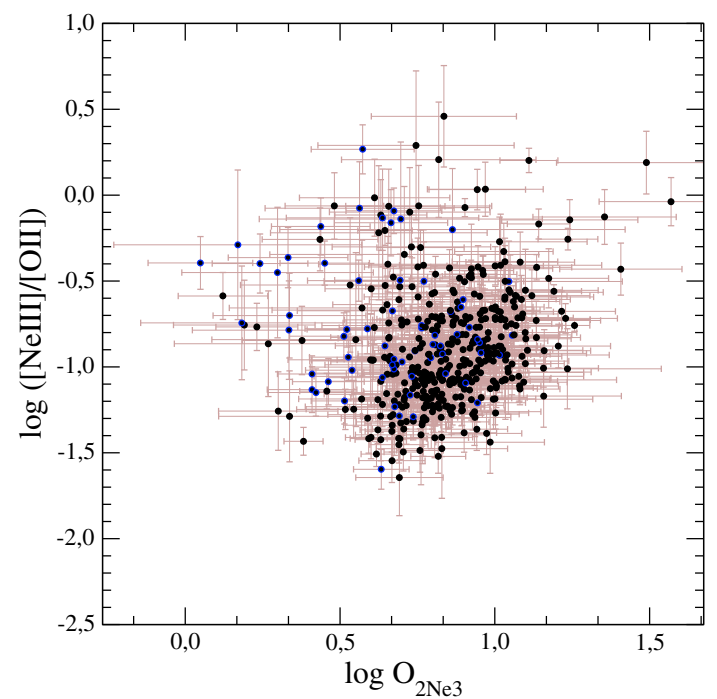

Fig. 6. Relation between the $\mathrm{O}_{2 \mathrm{Ne} 3}$ parameter and the emission-line ratio $[\mathrm{NeIII}] /[\mathrm{OII}]$ for the VVDS star-forming galaxies at $0.9<z<1.24$. $\mathrm{O}_{2 \mathrm{Ne} 3}$ has been calculated using either the flux of $\mathrm{H} \beta$ (blue squares), $\mathrm{H} \gamma$ (black squares), or $\mathrm{H} \delta$ (white squares).

The filled histogram represents the residuals in relation with the abundances using the same parameter, but with an average reddening correction for all the sample equivalent to $A_{V}=0.5 \mathrm{mag}$. On the other hand, the empty thick line histogram represents the residuals with the abundances deduced with the same parameter, but using equivalent widths and correction factors based on the shape of the continuum calculated in a similar way as in Kobulnicky \& Phililps (2003). As we can see, both the mean value of the residuals and the dispersion of the distribution are much better in the first case.

As described in Pérez-Montero et al. (2007), the $\mathrm{O}_{2 \mathrm{Ne} 3}$ parameter has the same problems than $\mathrm{O}_{23}$, the most important being its degeneracy with metallicity. This means that for a given value of the $\mathrm{O}_{2 \mathrm{Ne} 3}$ parameter we can find two possible values of the metallicity. Among the numerous calibrations of the $\mathrm{O}_{23}$ parameter there is always a calibration for high metallicities (upper branch) and another one for low metallicities (lower branch) (e.g. Skillman 1989). It is thus mandatory to be able to distinguish between these two metallicity regimes. The ratio $[\mathrm{NeIII}] /[\mathrm{OII}]$ has been proposed by Nagao et al. (2006) as an empirical calibrator of the metallicity but, due to its high dependence on ionization parameter, it does not allow to retrieve accurate determinations of $\mathrm{O} / \mathrm{H}$. However, it can be used to choose the appropriate branch (low or high metallicity regime) for the $\mathrm{O}_{2 \mathrm{Ne} 3}$ parameter. The results obtained by Pérez-Montero et al. (2007) indicate that galaxies with $\log ([\mathrm{NeIII}] /[\mathrm{OII}])<-1.0$ are in the high metallicity regime, although the contrary is uncertain. In Fig. 6, we show the relation between $\mathrm{O}_{2 \mathrm{Ne} 3}$ parameter and $\log ([\mathrm{NeIII}] /[\mathrm{OII}])$ for the VVDS galaxies harbouring the involved emission lines. We see that only a fraction of the objects lie in the high metallicity regime using this criterion. For the rest of galaxies with $\log ([\mathrm{NeIII}] /[\mathrm{OII}])>-1.0$, we constrain the metallicity regime by comparing the derived metallicities in low- and high-metallicity branches to the value derived with the Bayesian method described in Paper I or with the $\mathrm{O}_{23}$ parameter in the case of the redshift bin $0.5<z<0.9$. After this comparison we conclude that $95 \%$ of the sample of star-forming galaxies lie in the high metallicity regime. The selected objects have values of $\mathrm{O}_{2 \mathrm{Ne} 3}$ in the range $0.0-1.5$, corresponding to oxygen abundances in the range $8.0<12+\log (\mathrm{O} / \mathrm{H})<9.2$ in the upper branch.

In order to be consistent with metallicity estimates performed in Paper I, we use the calibrations of the upper branch of $\mathrm{O}_{23}$ parameter based on the models of Charlot \& Longhetti (2001). As it has been shown by Kewley \& Ellison (2008), it is very important to keep the consistency between the different empirical calibrators used to derive the metallicity of galaxies. If not, systematic differences as high as 0.7 dex can be observed for the $\mathrm{M}-\mathrm{Z}$ relation in the local universe as derived from the SDSS.

We check the consistency of this new method at higher redshift by comparing the metallicities derived using the $\mathrm{O}_{2 \mathrm{Ne} 3}$ and $\mathrm{O}_{23}$ parameters in the VVDS star-forming galaxies at $z<0.89$. Up to this redshift, all the emission lines required to compute these two parameters are observed in the VIMOS optical spectra. For this purpose we take into account only the oxygen abundances derived with an accuracy better than 0.3 dex. The results of this comparison are shown in Fig. 7. We distinguish three cases where $\mathrm{O}_{2 \mathrm{Ne} 3}$ parameter is estimated using $\mathrm{H} \beta$ (268 galaxies), $\mathrm{H} \gamma$ (217 galaxies) and $\mathrm{H} \delta$ (187 galaxies) respectively. Dashed lines represent the 1:1 relation while the solid lines are the best linear fit to the data. The best correlation is found in the first case, where the average of the residuals is 0.01 dex with a standard deviation of 0.17 dex. For the second case (middle panel) the mean value of the residuals is also 0.01 dex, but the standard deviation increases to 0.25 dex. Finally, in the third case (right panel), we obtain a mean value of the residuals of 0.04 dex and a standard deviation of 0.24 dex. The same kind of analysis comparing the metallicities derived from the $\mathrm{O}_{2 \mathrm{Ne} 3}$ parameter on one side and the Bayesian Method on the other side gives a mean value of the residuals of 0.03 dex only with a standard deviation of 0.31 dex.

\section{The mass-metallicity relation}

We study the M-Z relation for the redshift bin $0.89<z<1.24$, using the stellar masses and the metallicities derived in previous sections. We take only galaxies with a stellar mass higher than the limiting mass imposed in Fig. 4 for a mean redshift $z=1$, corresponding to a logarithmic stellar mass in solar masses of 8.9 for the deep fields and 9.6 for the wide fields. We take only galaxies with an uncertainty in the calculation of the oxygen abundance lower than 0.3 dex, so they remain 42 starforming VVDS galaxies: 27 in the deep fields and 15 in the wide fields. The galaxies are listed in Table 2 along with their $I_{\mathrm{AB}}$ apparent magnitude, spectroscopic redshift, and derived metallicities and stellar masses. In the case of metallicity, all the errors due to the uncertainties on the emission-line fluxes are lower than the intrinsic uncertainty of the empirical parameter based on [NeIII] emission lines, which is 0.2 dex for the calibration of the upper branch of the $\mathrm{O}_{2 \mathrm{Ne} 3}$ parameter (Pérez-Montero et al. 2007). The spectra of all these galaxies are available via the CENCOS database ${ }^{1}$. We have to notice that, after applying the selection criteria described above, no galaxies from the $10 \mathrm{~h}$ and 14h VVDS fields remain in the final sample.

In left panel of Fig. 8 we show the relation between stellar mass and oxygen abundance for these 42 galaxies. For comparison, we show also the $\mathrm{M}-\mathrm{Z}$ relation derived in the local universe $(z<0.3)$ by Tremonti et al. (2004) with SDSS data (solid line). Note that the comparison can be made directly as the same method/calibration (namely Charlot \& Longhetti 2001, see Sect. 2.3) has been used to derive metallicities in the local

\footnotetext{
1 http://cencosw.oamp.fr
} 


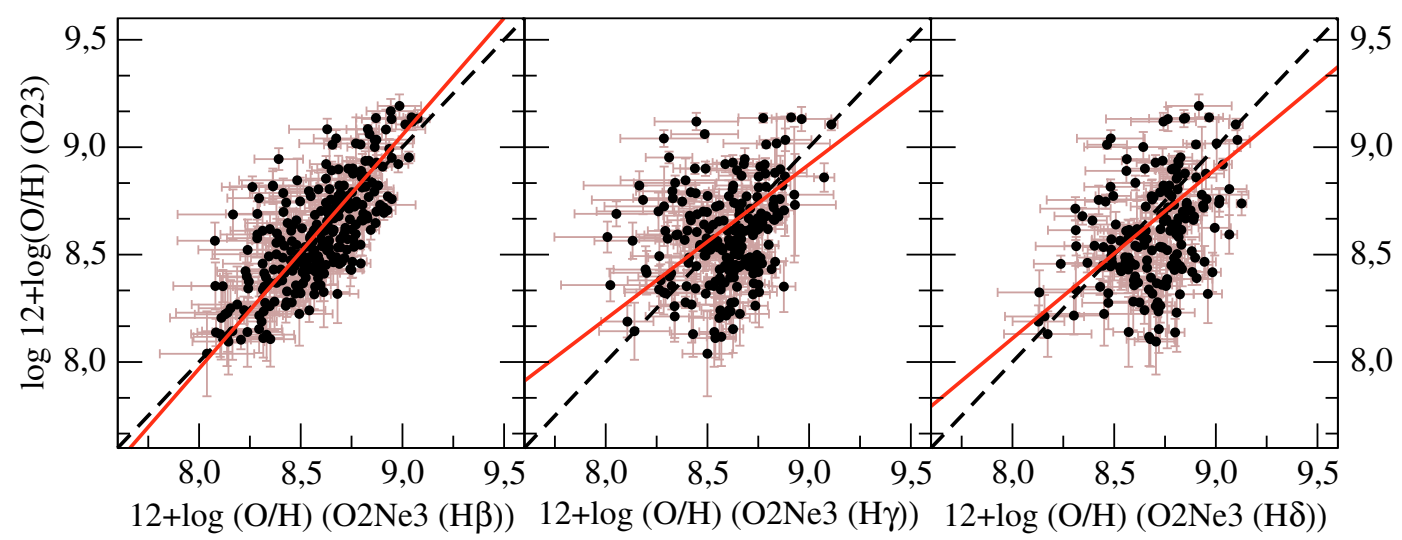

Fig. 7. Comparison between the oxygen abundances derived with the $\mathrm{O}_{23}$ and $\mathrm{O}_{2 \mathrm{Ne} 3}$ parameters. Three cases are distinguished: $\mathrm{O}_{2 \mathrm{Ne} 3}$ parameter is estimated using $\mathrm{H} \beta$ (left panel, 268 galaxies), $\mathrm{H} \gamma$ (middle panel, 217 galaxies) or $\mathrm{H} \delta$ (right panel, 187 galaxies) emission line. Only VVDS star-forming galaxies with an accuracy on $\mathrm{O} / \mathrm{H}$ lower than $0.3 \mathrm{dex}$ are considered. Dashed lines represent the 1:1 relation while the solid lines are the best linear fit to the data.

Table 2. Sample of 42 star-forming VVDS galaxies in the redshift regime $0.89<z<1.24$ with a determination of both the stellar mass and the oxygen abundance. We report also their spectroscopic redshift and $I$-band (AB system) apparent magnitude.

\begin{tabular}{|c|c|c|c|c|c|c|c|c|c|}
\hline Object & $\overline{I_{\mathrm{AB}}}$ & $\bar{z}$ & $12+\log (\mathrm{O} / \mathrm{H})$ & $\overline{l o g}\left(M_{*} / M_{\odot}\right)$ & Object & $\overline{I_{\mathrm{AB}}}$ & $\bar{z}$ & $=12+\log (\mathrm{O} / \mathrm{H})$ & $\overline{l o g}\left(M_{*} / M_{\odot}\right)$ \\
\hline VVDS000016597 & 23.24 & 0.9689 & 8.68 & $9.24 \pm 0.30$ & VVDS020386743 & 22.58 & 1.0465 & 8.91 & $9.43 \pm 0.11$ \\
\hline VVDS000025365 & 22.80 & 0.8956 & 8.50 & $10.10 \pm 0.25$ & VVDS020389342 & 23.76 & 0.9057 & 8.36 & $9.30 \pm 0.14$ \\
\hline VVDS000028748 & 21.24 & 0.9523 & 9.16 & $9.42 \pm 0.29$ & VVDS020460957 & 23.36 & 0.9615 & 8.96 & $9.34 \pm 0.08$ \\
\hline VVDS020098524 & 23.13 & 0.9044 & 8.68 & $8.95 \pm 0.07$ & VVDS020461893 & 23.45 & 1.0486 & 8.73 & $9.27 \pm 0.07$ \\
\hline VVDS020109569 & 22.50 & 1.0800 & 8.51 & $9.40 \pm 0.05$ & VVDS020462475 & 22.76 & 0.9626 & 8.35 & $9.36 \pm 0.07$ \\
\hline VVDS020127928 & 21.23 & 0.9610 & 8.89 & $10.43 \pm 0.07$ & VVDS220005349 & 22.36 & 0.9610 & 9.03 & $10.20 \pm 0.14$ \\
\hline VVDS020142000 & 22.16 & 0.9606 & 8.52 & $9.45 \pm 0.04$ & VVDS220077555 & 22.10 & 0.9825 & 8.25 & $9.89 \pm 0.07$ \\
\hline VVDS020150832 & 23.19 & 0.9628 & 8.38 & $9.16 \pm 0.06$ & VVDS220102663 & 21.83 & 1.1348 & 9.01 & $10.14 \pm 0.11$ \\
\hline VVDS020155109 & 22.17 & 0.9840 & 8.57 & $9.62 \pm 0.06$ & VVDS220108994 & 22.3 & 0.9007 & 8.16 & $9.36 \pm 0.11$ \\
\hline VVDS020165728 & 23.11 & 0.9051 & 8.62 & $9.06 \pm 0.05$ & VVDS220156608 & 22.06 & 0.9871 & 8.68 & $10.53 \pm 0.11$ \\
\hline VVDS020175617 & 23.18 & 0.9046 & 8.72 & $8.94 \pm 0.16$ & VVDS220294849 & 22.17 & 0.9933 & 8.65 & $9.93 \pm 0.17$ \\
\hline VVDS020196604 & 23.12 & 0.9607 & 8.60 & $9.06 \pm 0.14$ & VVDS220376206 & 21.85 & 1.2440 & 8.74 & $10.02 \pm 0.08$ \\
\hline VVDS020199214 & 22.62 & 0.9126 & 8.27 & $9.28 \pm 0.07$ & VVDS220380153 & 21.55 & 0.9332 & 8.72 & $9.94 \pm 0.06$ \\
\hline VVDS020201361 & 23.27 & 1.0572 & 8.83 & $9.03 \pm 0.14$ & VVDS220390996 & 21.76 & 0.9071 & 8.41 & $9.75 \pm 0.09$ \\
\hline VVDS020210745 & 23.97 & 0.8983 & 8.61 & $9.31 \pm 0.14$ & VVDS220457904 & 22.30 & 1.0666 & 8.48 & $10.06 \pm 0.08$ \\
\hline VVDS020247631 & 23.37 & 0.9171 & 9.04 & $9.03 \pm 0.10$ & VVDS220485833 & 20.99 & 0.9807 & 8.58 & $10.33 \pm 0.10$ \\
\hline VVDS020276040 & 21.71 & 0.9249 & 9.02 & $10.05 \pm 0.07$ & VVDS220535047 & 22.29 & 1.0159 & 8.67 & $10.01 \pm 0.10$ \\
\hline VVDS020294045 & 22.80 & 1.0019 & 8.29 & $9.17 \pm 0.06$ & VVDS220540780 & 21.91 & 0.9089 & 8.72 & $10.85 \pm 0.14$ \\
\hline VVDS020304000 & 23.46 & 0.9561 & 8.64 & $9.28 \pm 0.13$ & VVDS220559147 & 22.25 & 0.8984 & 8.99 & $10.48 \pm 0.14$ \\
\hline VVDS020322281 & 22.89 & 0.9320 & 8.65 & $9.32 \pm 0.07$ & VVDS220603467 & 21.85 & 0.9156 & 8.81 & $10.31 \pm 0.08$ \\
\hline VVDS020385905 & 22.36 & 0.9759 & 8.44 & $9.84 \pm 0.01$ & VVDS220605155 & 22.06 & 0.9519 & 8.52 & $9.78 \pm 0.07$ \\
\hline
\end{tabular}

and high-redshift galaxies. The respective limiting mass for both deep and wide fields are shown as vertical solid lines.

In order to test the global evolution in metallicity of starforming galaxies we make the same analysis carried out in Paper I, consisting in the calculation of the mean shift on metallicity by fitting the same curve deduced for the SDSS sample to our data points and, hence, the derivation of a different zeropoint. These fits are shown in left panel of Fig. 8 as a blue dashed-point line for the galaxies in the deep fields and a red dashed-point line for the wide field galaxies. The mean difference in metallicity found with respect to the local M-Z relation are $-0.29 \pm 0.21$ and $-0.12 \pm 0.15$ for the wide and deep field galaxies respectively. As it was shown in Paper I, we see a stronger evolution of the mean metallicity of galaxies in the wide sample. As the limiting mass of the wide sample is much higher than in the deep sample, this could be due to a more significant evolution in metallicity for the most massive galaxies. The M-Z relation derived in the redshift range $0.89<z<1.24$ is rather similar to the one derived in Paper I for the redshift bin $0.7<z<0.9$. However, this difference between the wide and deep samples could be due to different selection effects. First the exposure time was higher for the deep fields which could lead to a higher dispersion in the metallicity distribution of these objects, as the number of high-metallicity galaxies, i.e. with fainter emission lines, is larger. There is also a larger number of lowmass galaxies in the deep sample, for which the metallicity is also lower compared with the average value.

We assess also the shape of the $\mathrm{M}-\mathrm{Z}$ relation calculating a running median of the metallicity for the whole sample (both deep and wide fields) along the stellar mass axis in order to compare it with the results from Paper I and the relation deduced for the local universe using the SDSS sample (Tremonti et al. 2004). The method to calculate these median is identical to that described in Paper I: we have taken four bins in stellar mass, with a similar number of objects in each bin $(\approx 11)$ and then we calculate the median of 1000 bootstraps estimates. These 


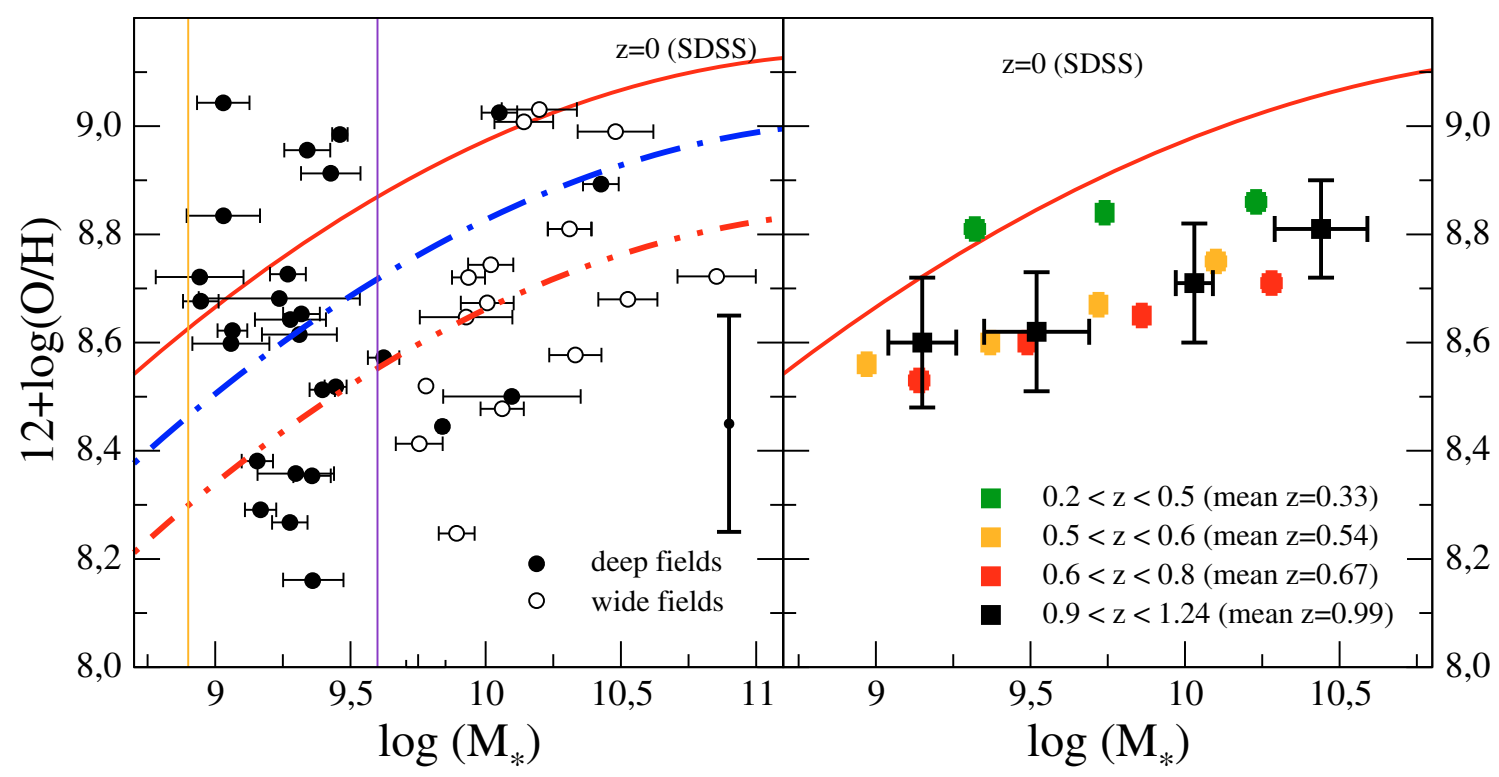

Fig. 8. Relation between the gas-phase metallicity and the stellar mass (in solar masses) for VVDS star-forming galaxies in the redshift range $0.89<z<1.24$. In left panel, we show the 42 individual VVDS star-forming galaxies (black circles: deep fields; white circles: wide fields). Vertical solid lines represent the corresponding limiting mass to the deep and wide samples. The solid red line represents the Tremonti et al. (2004) $\mathrm{M}-\mathrm{Z}$ relation for the SDSS sample. We show as well the typical uncertainty of the $\mathrm{O}_{2 \mathrm{Ne} 3}$ parameter as vertical error bars. The blue dashed-point curve represents the fit of the Tremonti curve to the VVDS galaxies of the deep sample, and the red for the wide sample. In right panel, we show the running medians of the whole sample (black squares, both deep and wide fields), as compared with the results from Paper I at lower redshifts (coloured squares).

medians are shown in the right panel of Fig. 8 as black squares. The error bars associated to the median metallicities of galaxies with $z>0.9$ are much higher than the ones derived in Paper I at lower redshifts, due to the lower number of objects in each mass bin. The M-Z relation found for $0.9<z<1.24$ is fully compatible with the results of Paper I for $z>0.5$ in absolute value and shape, pointing to a significant global chemical evolution for redshifts between 0 and 0.5 . However, for a redshift $z>0.5$, we do not find a significant global evolution within the error bars up to $z=1$. Regarding the shape of the $M-Z$ relation, we see a flattening as compared the with the $\mathrm{M}-\mathrm{Z}$ relation in the local universe. This could be a consequence of more effective yields for the more massive galaxies, where the evolution is stronger. However, again, we do not appreciate any significant evolution of the slope of the M-Z relation between redshifts 0.5 and 1 .

It is expected somehow that the sample of star-forming galaxies considered in this work can be affected by some selection effects which lead to a higher fraction of objects with low metallicities. Firstly, the selection criterion on [NeIII] emission lines biases the sample towards lower metallicities as pointed by Nagao et al. (2006) and Pérez-Montero et al. (2007). Secondly, the criterion on hydrogen recombination lines $(\mathrm{H} \gamma$ and $\mathrm{H} \delta)$ implies a sample of galaxies with higher star-formation efficiencies. According to Ellison et al. (2008), this leads also to galaxies with lower metallicities. Nevertheless, the consistency between the metallicities derived using the $\mathrm{O}_{2 \mathrm{Ne} 3}$ parameter in this redshift range and those at lower redshifts indicates that the probable selection effects inherent to our sample do not have a significant weight in the overall deduced metallicity.

\section{Summary and conclusions}

We have carried out a continuation of our study of the massmetallicity relation of star-forming VVDS galaxies extending our analysis up to redshift $z \sim 1.24$.
We selected galaxies with a reliable measurement of [OII], [NeIII] and $\mathrm{H} \gamma$ or $\mathrm{H} \delta$ emission lines and we identified starforming galaxies with the diagnostic diagram based on these lines and presented in Pérez-Montero et al. (2007). Then, we derived oxygen gas-phase abundances using the empirical parameter $\mathrm{O}_{2 \mathrm{Ne} 3}$. We have shown that these new methods are consistent with those based on [OIII] if we use the same calibrations. As it is based on weaker emission lines that, generally, are more affected by absorption effects, the only problem appears as a selection effect, biaising the sample towards galaxies with bright emission lines and, hence, towards lower metallicities. The relative number of narrow-line AGNs at higher redshifts is thus to be taken with caution.

The final sample used to derive the relation between metallicity and stellar mass consists of 42 VVDS star-forming galaxies with uncertainties lower than 0.3 dex in both oxygen abundance and stellar mass. These have been selected to be larger than the previously calculated limiting mass for this redshift range. Since the number of selected objects is sensibly lower than in our previous study (Paper I), the sample is not as statistically significant but we have found fully consistent results.

The zero-point and the slope of the $\mathrm{M}-\mathrm{Z}$ relation in the $0.9<z<1.24$ redshift range for both the deep and wide fields are rather similar to the values reported in Paper I for $z>0.5$, showing a significant global cosmic chemical evolution in relation with the local universe. We detect also a flattening of the $\mathrm{M}-\mathrm{Z}$ relation in comparison with the SDSS-based relation in the local universe (Tremonti et al. 2004). This evolution is stronger for the galaxies of the wide sample, consistently with the fact that most massive galaxies have undergone a stronger chemical evolution. The evolution of massive galaxies compared with the local sample can be understood as a consequence of lower effective yields for the most massive galaxies at larger redshifts, according to an hierarchical scenario of formation and evolution of galaxies. 
Nevertheless, despite the probable selection effects of the final sample, we do not appreciate any significant evolution neither in the absolute value of the metallicity nor in the shape of the $\mathrm{M}-\mathrm{Z}$ relation for redshifts larger than 0.5 . This can be a consequence of the smaller time interval in the redshift range $0.7<z<1$ combined with a larger uncertainty due to the lower number of objects with an appropriate signal-to-noise ratio for the used emission-lines. In any case, our results are fully compatible with those published for $z \approx 1$ in the DEEP2 sample (Shapley et al. 2005; Liu et al. 2008) or in the GOODS sample (Cowie \& Barger 2008), who find 0.2-0.3 dex lower metallicities in relation with the local universe.

Acknowledgements. We thank C. Tremonti for having made the original platefit code available to the VVDS collaboration. We also thank the referee, Roberto Maiolino, for many valuable suggestions and comments, which have helped us to improve this paper. This work has been partially supported by the CNRS-INSU and its Programmes Nationaux de Galaxies et de Cosmologie (France) and by project AYA-2004-08260-C03-03 of the Spanish National Plan for Astronomy and Astrophysics. The VLT-VIMOS observations have been carried out on guarateed time (GTO) allocated by the European Southern Observatory (ESO) to the VIRMOS consortium, under a contractual agreement between the Centre National de la Recherche Scientifique of France, heading a consortium of French and Italian institutes, and ESO, to design, manufacture and test the VIMOS instrument. Based on observations obtained with MegaPrime/MegaCam, a joint project of CFHT and CEA/DAPNIA, at the Canada-France- Hawaii Telescope (CFHT) which is operated by the National Research Council (NRC) of Canada, the Institut National des Science de l'Univers of the Centre National de la Recherche Scientifique (CNRS) of France and the University of Hawaii. This work is based in part on data products produced at TERAPIX and the Canadian Astronomy Data Centre as part of the Canada-France-Hawaii Telescope Legacy Survey, a collaboration project of NRC and CNRS.

\section{References}

Arnouts, S., Vandame, B., Benoist, C., et al. 2001, A\&A, 379, 740

Brinchmann, J., Charlot, S., White, S. D. M., et al. 2004, MNRAS, 351, 1151

Brodie, J. P., \& Huchra, J. P. 1991, ApJ, 379, 157

Bruzual, G., \& Charlot, S. 2003, MNRAS, 344, 1000

Charlot, S., \& Longhetti, M. 2001, MNRAS, 323, 887

Cowie, L. L., \& Barger, A. J. 2008 [arXiv:0806.3457]

Ellison, S. L., York, B. A., Murphy, M. T., et al. 2008, MNRAS, 383, 30

Erb, D. K., Shapley, A. E., Pettini, M., et al. 2006, ApJ, 644, 813

Gallazzi, A., Charlot, S., Brinchmann, J., White, S. D. M., \& Tremonti, C. A. 2005, MNRAS, 362, 41

Garnett, D. R., Shields, G. A., Skillman, E. D., Sagan, S. P., \& Dufour, R. J. 1997, ApJ, 489, 63

Gavignaud, L., Bongiorno, A., Paltani, S., et al. 2006, A\&A, 457, 79

Giavalisco, M., Ferguson, H. C., Koekemoer, A. M., et al., 2004, ApJ, 600, L93

Iovino, A., McCracken, M. J., Garilli, B., et al. 2005, A\&A, 442, 423

Kauffmann, G., Heckman, T. M., Tremonti, C., et al. 2003, MNRAS, 341, 33

Kewley, L. J., \& Ellison, S. L. 2008, AJ, 801, accepted [arXiv:0801.1849]

Kobulnicky, H. A., \& Phillips, A. C. 2003, ApJ, 599, 1006

Lamareille, F., Mouhcine, M., Contini, T., Lewis, I., \& Maddox, S. 2004, MNRAS, 350, 396

Lamareille, F., Contini, T., Le Borgne, J.-F., et al. 2006, A\&A, 417, 839 Lamareille, F., Brinchmann, J., Contini, et al. 2009, A\&A, 495, 53 (Paper I)

Le Fèvre, O., Meilleur, Y., McCracken, M. J., et al. 2004, A\&A, 417, 839

Le Fèvre, O., Vettolani, G., Garilli, B., et al. 2005, A\&A, 439, 845

Le Fèvre, O., Vettolani, G., Garilli, B., et al. 2006, ApJ, 647, 790

Lequeux, J., Peimbert, M., Rayo, J. F., Serrano, A., \& Torres-Peimbert, S. 1979, A\&A, 80, 155

Liu, X., Shapley, A. E., Coil, A. L., Brinchmann, J., \& Ma, C.-P. 2008, ApJ, 678, 758

Maiolino, R., Nagao, T., Grazian, A., et al. 2008, A\&A, 488, 463

McCracken, H. J., Radovich, M., Bertin, E., et al. 2003, A\&A, 410, 17

Nagao, T., Maiolino, R., \& Marconi, A., 2006, A\&A, 459, 85
Pérez-Montero, E., Hägele, G. F., Contini, T., \& Díaz, A. I. 2007, MNRAS, 381, 125

Pilyugin, L. S., \& Ferrini, F. 2000, A\&A, 358, 72

Radovich, M., Arnaboldi, M., Ripepi, V., et al. 2004, A\&A, 417, 51

Richer, M. G., \& McCall, M. L. 1995, ApJ, 445, 642

Rola, C. S., Terlevich, E., \& Terlevich, R. J. 1997, MNRAS, 289, 419

Salim, S., Charlot, S., Rich, R. M., et al. 2005, ApJ, 619, L39

Savaglio, S., Glazebrook, L., Le Borgne, D., et al. 2005, ApJ, 635, 260

Scodeggio, M., Franzetti, P., Garilli, B., et al. 2005, PASP, 117, 1284

Shapley, A. E., Coil, A. L., Ma, C.-P., \& Bundy, K. 2005, ApJ, 635, 1006

Skillman, E. D. 1989, ApJ, 347, 883

Skillman, E. D., Kennicutt, R. C., \& Hodge, P. W. 1989, ApJ, 347, 875

Spergel, D. N., Verde, L., Peiris, H. V., et al. 2003, ApJS, 148, 175

Temporin, S., Iovino, A., Bolzonella, M., et al. 2008, A\&A, 482, 81

Tremonti, C. A., Heckman, T. M., Kauffmann, G., et al. 2004, ApJ, 613, 898

Warren, S. J., Cross, N. J. G., Dye, S., et al. 2007,

Zaritsky, D., Kennicutt, R. C., \& Huchra, J. P. 1994, ApJ, 420, 87

1 Laboratoire d'Astrophysique de Toulouse-Tarbes, Université de Toulouse, CNRS, Observatoire Midi-Pyrénées, 14 Av. E. Belin, 31400, Toulouse, France

e-mail: enrique.perez@ast.obs-mip.fr

2 IASF-INAF, via Bassini 15, 20133, Milano, Italy

3 INAF-Osservatorio Astronomico di Bologna, via Ranzani 1, 40127, Bologna, Italy

4 IRA-INAF, via Gobetti 101, 40129, Bologna, Italy

5 INAF-Osservatorio Astronomico di Capodimonte, via Moiariello 16, 80131, Napoli, Italy

6 Università di Bologna, Dipartimento di Astronomia, via Ranzani 1, 40127, Bologna, Italy

7 Laboratoire d'Astrophysique de Marseille, UMR 6110 CNRSUniversité de Provence, 38 rue Frederic Joliot-Curie, 13388 Marseille Cedex 13, France

8 Max Planck Institut für Astrophysik, 85741, Garching, Germany

9 INAF-Osservatorio Astronomico di Brera, via Brera 28, 20021, Milan, Italy

10 Institut d'Astrophysique de Paris, UMR 7095, 98 bis Bvd Arago, 75014, Paris, France

11 Observatoire de Paris, LERMA, 61 Avenue de l'Observatoire, 75014, Paris, France

12 Astrophysical Institute Potsdam, An der Sternwarte 16, 14482, Potsdam, Germany

13 INAF-Osservatorio Astronomico di Roma, via di Frascati 33, 00040, Monte Porzio Catone, Italy

14 Universitá di Milano-Bicocca, Dipartimento di Fisica, Piazza delle Scienze 3, 20126, Milano, Italy

15 Integral Science Data Centre, ch. d'Écogia 16, 1290, Versoix, Switzerland

16 Geneva Observatory, ch. des Maillettes 51, 1290, Sauverny, Switzerland

17 Astronomical Observatory of the Jagiellonian University, ul Orla 171, PL-30-244, Kraków, Poland

18 Centre de Physique Théorique, UMR 6207 CNRS-Université de Provence, 13288, Marseille, France

19 Centro de Astrofísica da Universidade do Porto, Rua das Estrelas, 4150-762, Porto, Portugal

20 Institute for Astronomy, 2680 Woodlawn Dr., University of Hawaii, Honolulu, Hawaii, 96822, USA

21 School of Physics \& Astronomy, University of Nottingham, University Park, Nottingham, NG72RD, UK

22 Max Planck Institut für Extraterrestrische Physik (MPE), Giessenbachstrasse 1, 85748 Garching bei München, Germany

23 Canada France Hawaii Telescope corporation, Mamalahoa Hwy, Kamuela, 96743, USA 